\title{
CHEMICAL SEPARATION VS ROTATION IN A AND F-STARS
}

\author{
Paul CHARBONNEAU High Altitude Observatory, National Center \\ for Atmospheric Research ${ }^{1}$, P.O. Box 3000, Boulder, CO 80307, U.S.A.
}

\begin{abstract}
The role played by rotationally-induced mixing in the diffusionbased models for non-magnetic chemically peculiar stars is investigated. This paper focuses on one specific rotationally controlled mixing mechanism, namely thermally-driven meridional circulation. Its effects on the time evolution of chemical abundances are illustrated by means of three specific examples. The first two concern the diffusion model for FmAm stars, where it is shown that while circulation has a determining influence on the settling of Helium, it has no significant effect on the diffusion of heavier metals once the He superficial convection zone has disappeared. The third example is concerned with the diffusion/mass loss model for $\lambda$ Bootis stars. It is shown that the inclusion of circulation prevents the appearance of generalized underabundances at any epoch of the evolution, indicating that the diffusion/mass loss model for these objects must be abandoned.
\end{abstract}

Keywords: Abundances; Diffusion; Rotation

\section{INTRODUCTION: PARTICLE TRANSPORT IN STARS}

The chemical abundances observed in the photosphere of a given star at a given time are of course function of the initial abundances in the interstellar cloud from which that star formed, but are also affected by a number of physical mechanisms having operated with various efficiencies in the course of its evolution to the present time. If one were to draw a list of physical mechanisms susceptible to alter the chemical abundances in the outer envelope, such a list would rapidly become very long. It should include the following:

(1) Microscopic diffusion

(2) Gravitational settling / differential thermal diffusion

(3) Diffusion via differential radiative acceleration

(4) Thermal convection (superadiabatic medium)

(5) Meridional circulation

(6) Convective overshoot

(7) Mass Loss / Accretion

(8) Convection in magnetically-induced thermal shadows

(9) Turbulence via shear flow instabilities

(10) Turbulence via baroclinic instabilities

Mechanisms (1), (2) and (3) are the processes leading to chemical separation (see e.g. Michaud, these proceedings). These are basic physical mechanisms that cannot be omitted a priori in modelling the evolution of abundances.

1 The National Center for Atmospheric Research is sponsored by the National Science Foundation. 
Mechanisms (5), (9) and (10) are driven and/or controlled by rotation. In principle, all ten of these mechanisms (and a few more!) should be included in a truly self-consistent model of chemical evolution for chemically peculiar stars. In practice, this is a overwhelming task, complicated by the fact that many of these mechanisms, especially as one moves down the list, are poorly understood. Consequently, their effects on particle transport are often impossible to model without the introduction of free parameters.

The approach favored here is to include in transport calculations all mechanisms that are well understood and whose effects can be modeled without free parameters. Only if the resulting particle transport model fails to reproduce observations are additional mechanisms included. This not only yields maximally deterministic models for chemically peculiar (CP) stars, but also allows the use of observed abundances to constrain parameterizations of other transport mechanisms, such as mass loss, turbulence, etc. In this way, observed abundances become probes of stellar interior hydrodynamics.

Accordingly, this review paper focuses on the effects of rotationally-induced meridional circulation, in the framework of diffusion models for non-magnetic chemically peculiar stars. This is done in the form of three "case studies", the first two in the context of the diffusion model for FmAm stars ( $\$ 3)$, and the third in that of diffusion model(s) for $\lambda$ Bootis stars (\$4). But first, a rapid look at the origin and characteristics of meridional circulation is required, and is the topic of the following section.

\section{MERIDIONAL CIRCULATION}

As such, the term "meridional circulation" is applicable to any large-scale fluid motion in meridional $(r, \theta)$ planes. This may refer to mechanically-driven spinup/spin-down flows such as Ekman circulation, flows in convection zones driven by anisotropies in turbulent stresses caused by the action of Coriolis forces on turbulent eddies (such as that observed in the solar convection zone), flows induced by gravitational distorsions due to the presence of a binary component, and so on. There are also a variety of thermally driven flows, the better understood of which is the flow driven by thermal imbalance on isobaric surfaces in a rotating star (see e.g., Tassoul 1978, chap. 7 and 8). It is the effect of the latter specific "meridional circulation" that will be investigated in the following sections. A closer look at its physical origin is therefore in order.

\subsection{Thermally-driven meridional circulation}

Consider the momentum and energy equations (with no magnetic fields and neglecting viscosity):

$$
\begin{gathered}
\frac{\partial \mathbf{v}}{\partial t}+(\mathbf{v} \cdot \nabla) \mathbf{v}=-\frac{1}{\rho} \nabla p-\mathbf{g}, \\
\rho c_{v}\left(\frac{\partial T}{\partial t}+\mathbf{v} \cdot \nabla T\right)+p \nabla \cdot \mathbf{v}=\rho \epsilon_{N U C}-\nabla \cdot \mathcal{F},
\end{gathered}
$$

where $\mathbf{v}$ is the total velocity, and with the radiative flux $\mathcal{F}$ given by

$$
\mathcal{F}=-\frac{4 a c T^{3}}{3 \kappa \rho} \nabla T
$$


In a static $(\mathbf{v}=0)$ steady-state $(\partial / \partial t=0)$ configuration, and outside of the nuclear burning region $\left(\epsilon_{N U C}=0\right)$, these reduce to the familiar

$$
\begin{aligned}
& \nabla p=-\rho \mathbf{g}, \\
& \nabla \cdot \mathcal{F}=0 .
\end{aligned}
$$

Consider now an axisymmetric rotating configuration and write

$$
\mathbf{v}=\mathbf{u}+\Omega \varpi \hat{\mathbf{e}}_{\phi} . \quad(\varpi=r \sin \theta)
$$

The meridional components of the momentum equation become (in cylindrical coordinates):

$$
\frac{\partial \mathbf{u}}{\partial t}+(\mathbf{u} \cdot \nabla) \mathbf{u}=-\frac{1}{\rho} \nabla p-\mathbf{g}+\Omega^{2} \varpi \hat{\mathbf{e}}_{\varpi},
$$

It can be shown that a steady-state in strict hydrostatic and radiative equilibrium cannot be constructed in this case. More specifically, hydrostatic solutions satisfying mechanical equilibrium in general do not satisfy $\nabla \cdot \mathcal{F}=0$. This is due to the centrifugal term in (5), that introduces departures from spherical symmetry. As a consequence, the outward transport of energy is no longer isotropic, leading to the growth of temperature difference along isobaric surfaces. These lead to pressure perturbations that destroy what used to be a mechanical equilibrium state. This is the Von Zeipel paradox (see e.g. Tassoul 1978, §7.2). The fundamental constraint, however, is not satisfying eq. (3b), but rather to conserve energy [i.e., satisfying eq. (1b)] ! One possibility is to abandon the hypothesis of strict hydrostatic equilibrium, by allowing for the existence of a large-scale meridional flow such that

$$
\rho c_{v}(\mathbf{u} \cdot \nabla) T+p \nabla \cdot \mathbf{u}=-\nabla \cdot \mathcal{F} .
$$

The task is then to compute the velocity field $\mathbf{u}$ required to balance out the non-vanishing $\nabla \cdot \mathcal{F}$ term. This is nowhere as straightforward as it may appear, since $\mathbf{u}$ will also make contributions on the LHS of the momentum equations. In particular, the presence of a large-scale meridional velocity field leads to the advection of angular momentum. The ensuing local variations in angular velocities then feed back on the pressure and density stratification, leading to further temperature variations along isobaric surfaces. But we initially introduced $\mathbf{u}$ in the energy equation precisely to balance out these temperature variations! The presence of a large-scale meridional velocity field thus introduces additional (non-linear and two-way) coupling between the various governing equations, making self-consistent computations of $\mathbf{u}$ extremely arduous. Although solutions have been obtained, this is still an active area of research in stellar fluid dynamics.

\subsection{The Tassoul \& Tassoul (1982) solutions}

Tassoul \& Tassoul (1982; see also Tassoul 1990, and references therein) have obtained self-consistent meridional circulation solutions based on power serie expansions in $\epsilon=\Omega^{2} R^{3} / G M$ (the ratio of centrifugal to gravitational force at the equator). They assume that the advection of angular momentum by circulation is balanced by enhanced viscous action due to small-scale turbulence. They 
ascribe the origin of the latter to baroclinic instabilities in the radiative envelope. The required level of (isotropic) turbulence is moderate; they use a turbulent viscosity $\nu_{T}=10^{3}-10^{6} \nu_{m}$, where $\nu_{m}$ is the microscopic viscosity. The circulation pattern that they obtain is characterized by a single cell, with the flow rising in polar regions and sinking near the equator (see e.g. Fig. 3 and 4 of Tassoul \& Tassoul 1982). Their circulation velocities are proportional to $v_{e}^{2}$, where $v_{e}$ is the equatorial surface rotational velocity. In the outer part of the radiative envelope of a $2 M_{\odot}$ star rotating with $v_{e}=100 \mathrm{~km} \mathrm{~s}^{-1}$, one has $\|\mathrm{u}\| \sim 10^{-3} \mathrm{~cm} \mathrm{~s}^{-1}$. In the outer layers of the star, the vertical component of the circulation velocity is nearly independent of depth, except within a thin boundary layer below the surface. The circulation patterns used in the transport calculations presented below are computed using this formalism.

\subsection{The Zahn (1992) model}

Recently, Zahn (1992) has obtained meridional circulation solutions valid for all rotation rates, but only in the special case of strong horizontal turbulence $\left(\nu_{T, H} \sim\right.$ $10^{10} \nu_{m}$ ). The circulation patterns he obtains are controlled mostly by variations in angular velocity gradient associated with angular momentum loss due to braking of the outer layers by a magnetically-confined wind (see also Sakurai 1991). As a consequence, the circulation patterns are time-dependent, and inspection of the mathematical expressions he derives for the radial component of the circulation velocity suggests that more than one circulation cell may exist. He also argues that in the case of a star not subjected to magnetic braking which is the case for $\mathrm{A}$ and early $\mathrm{F}$ stars-, the star rapidly reaches a circulationfree state. While no calculations of particle transport using Zahn's meridional circulation solutions have been carried out as yet, preliminary (and admittedly crude) estimates indicate that his circulation/horizontal turbulence model will be hard to reconcile with the diffusion-based models of CP stars. Nevertheless, efforts in that direction are certainly warranted.

\subsection{Circulation and particle transport}

Assuming that the form of the circulation velocity field is known, the next step is to incorporate it in particle transport models. The transport equation for the concentration $(c)$ of a chemical specie present in trace quantities in the envelope is:

$$
\rho \frac{\partial c}{\partial t}=\nabla \cdot(\rho D \nabla \ln c)-\rho(\mathbf{U}+\mathbf{V}) \cdot \nabla c-[\nabla \cdot(\rho \mathbf{V})+\rho S] c,
$$

where $D$ is the total diffusion coefficient, $\mathrm{U}$ is the velocity field associated with meridional circulation, $\mathbf{V}$ a velocity field including contributions from the processes leading to chemical separation (see e.g. Michaud 1987, §3), and $S$ a sink term corresponding to nuclear burning (important for the light elements $\mathrm{Li}$, $\mathrm{Be}, \mathbf{B}$, which get destroyed at relatively low temperatures via proton capture reaction). Mass loss, if present, can be incorporated as an extra contribution to U (see Charbonneau 1992c).

Equation (7) is essentially a conservation statement for the mass of the contaminant under study. One may note that circulation can only advect preexisting concentration gradients. This means that in a chemically homogeneous star, and in the absence of chemical separation and nuclear burning, circulation 
has no effect on abundances! However, in a more realistic situation, circulation does affect abundances, because it impedes chemical separation and advects to the surface abundance gradients set up by nuclear burning deeper in the envelope. The following sections present three illustrative examples of this influence, in the context of the diffusion model for chemically peculiar $A$ and $F$ stars.

\section{CHEMICAL SEPARATION IN FmAm STARS}

FmAm stars are main-sequence, pop. I, non-magnetic chemically peculiar mainsequence stars (see, e.g., Preston 1974). They are found in the effective temperature interval $7000 \lessgtr T_{\text {eff }} \lessgtr 10000 \mathrm{~K}$, and account for about $20 \%$ of all pop. I main-sequence stars in that $T_{\text {eff }}$ interval. They all appear to be slowly rotating $\left(v \cdot \sin i \leqslant 100 \mathrm{~km} \mathrm{~s}^{-1}\right)$. In fact, it seems that in this region of the HR diagram, slow rotation is a necessary and sufficient condition for the appearance of the FmAm abundance signature (Abt \& Levy 1985). The latter is characterized by underabundances of $\mathrm{Ca}$ and $\mathrm{Sc}$, by factors in the range 3100 , and strong overabundances of heavy elements, by factors $50-500$ with some definite trends with effective temperature and probably age (see Cayrel et al. 1991, and references therein; Burkhart \& Coupry 1991; Berthet 1992).

\subsection{The diffusion-based model}

The standard diffusion model for FmAm stars (Michaud et al. 1983; see also Charbonneau \& Michaud 1991) poses that FmAm stars are otherwise normal A and F stars whose portion of the superficial convection zone (CZ) associated with the first ionization of He has disappeared following the gravitational settling of He. Chemical separation then occurs up to the base of the hydrogen superficial $\mathrm{CZ}$, leading to strikingly different abundance patterns than in a case where chemical separation operates only up to the base of the (combined) heliumhydrogen $\mathrm{CZ}^{2}$ (see Fig. 1). The disappearence of the $\mathrm{He} \mathrm{CZ}$ then acts as a trigger to the development of the FmAm phenomenon. This, in turn, is possible only in the slower rotating stars, where circulation is too weak to sufficiently impede He settling.

The model is impressively succesful, in that (1) it predicts the correct abundance patterns, (2) it predicts the correct cutoff rotational velocity, and (3) by introducing mass loss (at a rate of order $10^{-15} M_{\odot} y r^{-1}$ ), it quantitatively

${ }^{2}$ For main-sequence A stars, application of the mixing length theory of convection yields an outer envelope structure where two distinct convectively unstable layers exist, the first (deeper) due to the first ioniztion of $\mathrm{He}$, and the second to the ionization of $H$. Estimates of convective overshoot efficiency suggest that the convectively stable layer located in between these two thin convection zones is thoroughly mixed (Latour, Toomre \& Zahn 1981). This is confirmed by numerical simulations (Sofia \& Chan 1984; Holweger, these proceedings). Consequently, it is common practice within the framework of diffusion models for chemically CP to assume that the whole region extending from the base of the $\mathrm{He}$ (lower) $\mathrm{CZ}$ all the way to the photosphere is thoroughly mixed. 

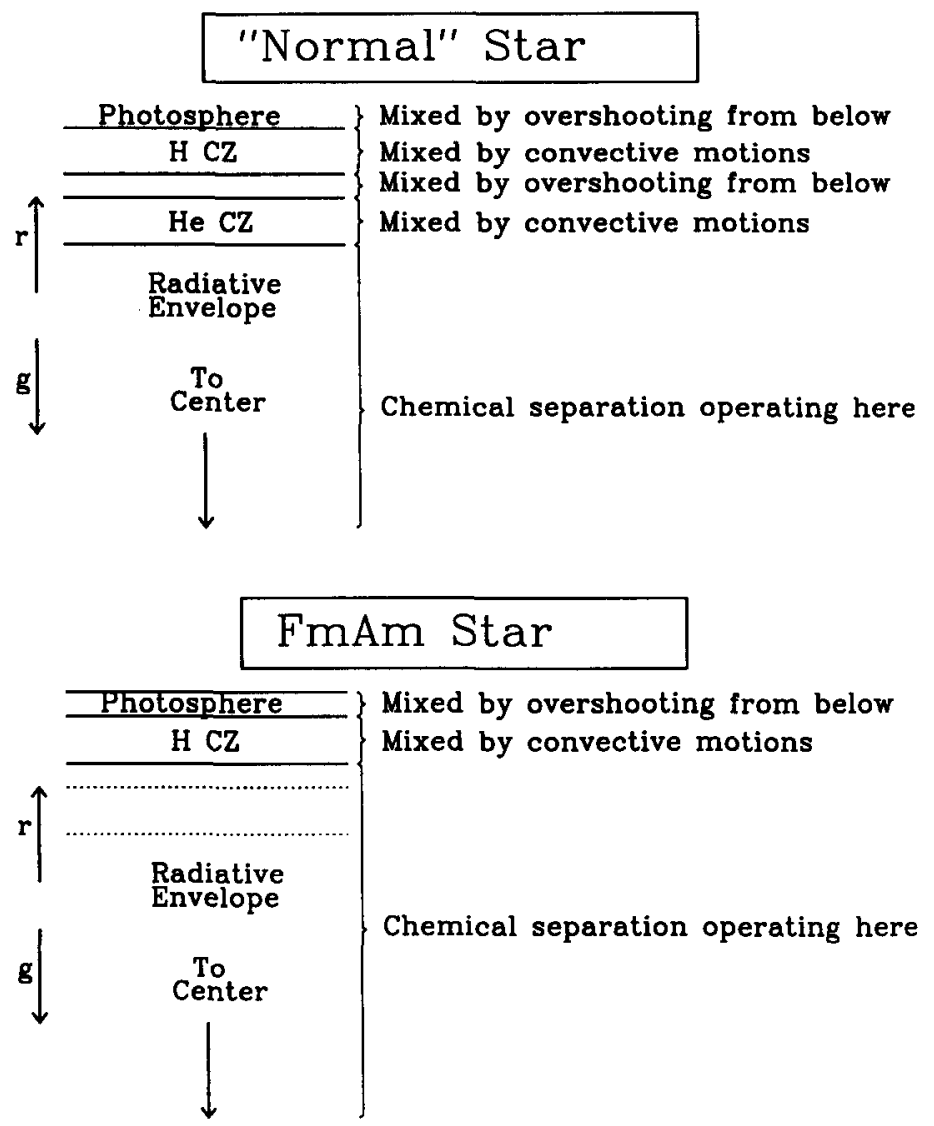

Figure 1 : Schematic representation of the outer structure of a "normal" A-star (top) and Am star (bottom). Note that the former has two distinct superficial convection zones, due to the first ionization of He (lower zone) and ionization of $\mathrm{H}$ (upper zone). The latter, however, has only the upper zone left, following gravitational settling of $\mathrm{He}$ from the outer layers. If the He abundance is solar at $t=0$, this requires a decrease by a factor of about 2.5 in the He content of the lower CZ.

reproduces anomalies for most elements. Note that in terms of (1) and (2), the model is fully deterministic (i.e., it involves no adjustable parameter).

The diffusion-based model of FmAm stars is, at this time, the leading contender among theoretical models for these objects. Some questions nevertheless remain: (1) What is the combined effect of circulation and mass loss on $\mathrm{He}$ settling and on the diffusion of heavy elements ? (2) How would evolutionary effects modify the abundance patterns predicted by the diffusion model ? (3) What is the driving mechanisms of mass loss? Point (1) is a matter of internal self-consistency, while (2) and (3) are rather fundamental physical issues. The remainder of this sections focuses on the former. 


\subsection{Circulation and Helium settling}

Using an approximate 1-D transport formulation and the Tassoul \& Tassoul (1982) meridional circulation solutions, Michaud (1982) showed that if meridional circulation is indeed the mechanism competing with the gravitational settling of He, then the observed $v \cdot \sin i$ cutoff observed in FmAm (and HgMn) stars is reproduced naturally, i.e., without adjustable parameters. This impressive agreement was confirmed by the 2-D computations of Charbonneau \& Michaud (1988). Charbonneau \& Michaud (1991) further went on to show that the requirement of $\mathrm{He}$ settling in stars rotating with $v_{e} \simeq 100 \mathrm{~km} \mathrm{~s}^{-1}$ translates into a very tight constraint on the strength of any turbulence existing in the upper part of the radiative envelope, indicating that He settling is rather sensitive to any additional mixing below the $\mathrm{CZ}$. On a different but related front, Michaud et al. (1983) found it necessary to introduce mass loss, at the rate of $10^{-15} M_{\odot} y r^{-1}$, in order to reproduce quantitatively the observed anomalies of metals in FmAm stars. One may then ask whether or not this additional transport mechanism will perturb the aforementionned agreement between the observed and predicted $v \cdot \sin i$ cutoff obtained in the absence of mass loss. The answer is no, as shown on Table 1. Each entry correspond to the time (in Myr) required for the disappearance of the $\mathrm{He} \mathrm{CZ}$, as a function of equatorial rotational velocity (lines, in $k m s^{-1}$ ) and mass loss rate (columns, in $M_{\odot} y r^{-1}$ ). Although larger mass loss rates do make He settling impossible in stars rotating below the observed cutoff, at $\dot{M}=10^{-15} M_{\odot} y r^{-1}$ the only effect is to slightly delay the disappearance of the $\mathrm{He} \mathrm{CZ}$ in the more rapidly rotating models, as compared to models without mass loss (first column). However, note how at $\dot{M} \gtrsim 10^{-13} M_{\odot} y r^{-1}$, mass loss by itself is sufficient to prevent the disappearance of the He CZ.

\section{Table 1}

Time required for the disappearance of the $\mathrm{He} \mathrm{CZ}\left(T_{e f f}=8000 \mathrm{~K}, \log g=4.3\right)$

\begin{tabular}{llllll}
\hline$v_{e}$ & 0 & $10^{-15}$ & $3 \times 10^{-15}$ & $10^{-14}$ & $10^{-13}$ \\
\hline \hline 0 & 1.0 & 1.0 & 1.25 & 4.25 & - \\
50 & 1.0 & 1.0 & 1.3 & 5.25 & - \\
75 & 1.25 & 1.35 & 1.75 & - & - \\
90 & 2.0 & 3.0 & $>20$ & - & - \\
\hline
\end{tabular}

\subsection{Circulation and metal abundances}

In the case of $\mathrm{He}$ settling, it was found that meridional circulation was the dominating mechanism competing with chemical separation. Yet Michaud et al. (1983), while not taking circulation into account in attempting to reproduce the abundance anomalies of metals, found it necessary to introduce instead a small mass loss. Furthermore, little, if any, correlation is observed in FmAm stars between $v \cdot \sin i$ 's and the amplitudes of abundance anomalies. This suggests 

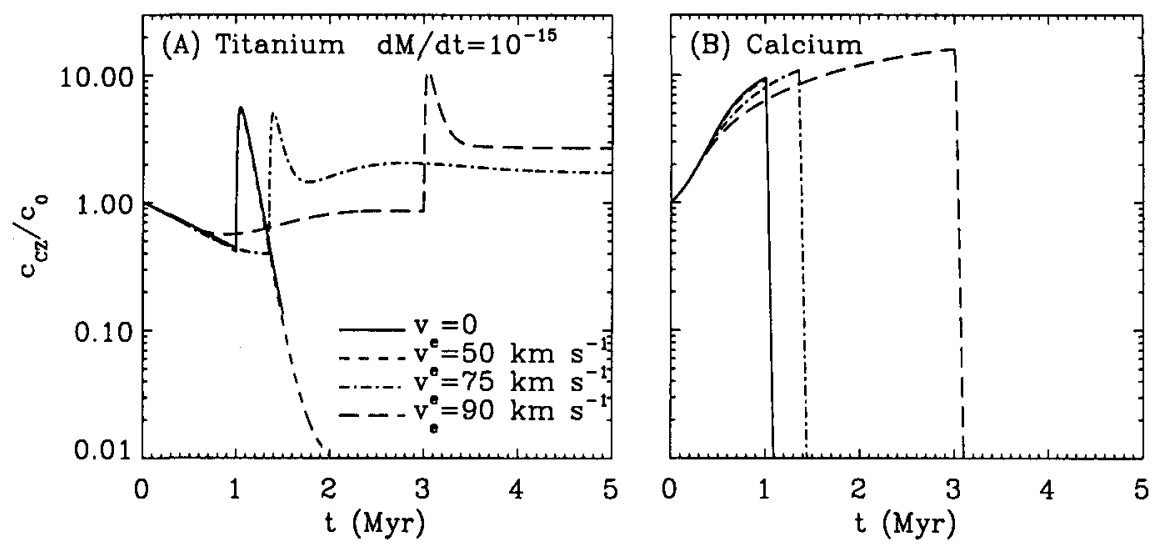

Figure 2 : Surface abundance evolution of calcium and titanium in a $T_{\text {eff }}=8000 \mathrm{~K}$ main sequence star losing mass at the rate $10^{-15} M_{\odot} y r^{-1}$, for various equatorial rotational velocities. The near discontinuities in the abundance curves correspond to the disappearance of the $\mathrm{He}$ $\mathrm{CZ}$, which occurs at various times depending on the adopted rotation and accretion rates (see Table 1 ).

that circulation influences very little the diffusion of metals. It may seem contradictory that circulation be so important for one aspect of the diffusion model, and negligible in the other.

As a first step in addressing this apparent paradox, Charbonneau \& Michaud (1991) obtained a large set of numerical solutions to chemical separation of metals in the presence of meridional circulation, but without mass loss. They showed that even at $v_{e}=100 \mathrm{~km} \mathrm{~s}^{-1}$, meridional circulation has little influence on the abundance evolution of metals once the $\mathrm{He} C Z$ has disappeared via settling. This is in general true even for elements such as $\mathrm{Ca}$ and Sc, that are not supported $\left(g_{R A D}<g\right)$ at the base of the $\mathrm{H} \mathrm{CZ}$. This is due to the fact that chemical separation velocities increase more rapidly, as one approaches the stellar surface from below, than do circulation velocities. Whereas the latter can dominate particle transport at the base of the $\mathrm{He} \mathrm{CZ}$, they become much smaller than typical chemical separation velocities at the base of the $\mathrm{H} \mathrm{CZ}$. The velocities associated with gravitational settling, for example, vary roughly as $T^{3 / 2} / \rho$; in the $T_{\text {eff }}=8000 \mathrm{~K} \log g=4.3$ main-sequence model used here, this corresponds to a change by a factor of 3 between the base of the $\mathrm{He} C Z$ and that of the $\mathrm{H} \mathrm{CZ}$, while circulation velocities remain essentially constant in that depth interval. The change is even larger for the velocities associated with differential radiative acceleration, since $g_{R A D}$ typically increases rapidly outward (see e.g. Michaud et al. 1976).

Figure 2 shows the surface abundance evolution predicted at early epochs by the diffusion model for $\mathrm{Ti}$ and $\mathrm{Ca}$, in a $T_{\text {eff }}=8000 \mathrm{~K}$, main-sequence stellar model. Both mass loss and circulation are included in the transport calculations. The abundance evolution is divided in two phases; a first phase where chemical separation operates only to the base of the $\mathrm{He} \mathrm{CZ}$, and a second phase where it operates all the way to the base of the $\mathrm{H} \mathrm{CZ}$. The transition from the first to the second phase coincides with the disappearance of the lower $\mathrm{CZ}$ due to 
He settling, which occurs after a time interval that is a function of the assumed rotational velocity (cf. Table 1 ). For $\mathrm{Ti}$, this transition shows up as a peak in the surface abundances, and for $\mathrm{Ca}$ as a sharp drop from a state of moderate overabundances to large underabundances. These occur because the radiative accelerations are markedly different at the bases of the $\mathrm{He}$ and $\mathrm{H} \mathrm{CZs;} \mathrm{Ti}$ is almost but not quite supported $\left(g_{R A D} \leqslant g\right.$ ) at the base of the He CZ, but is strongly supported $\left(g_{R A D} \gg g\right.$ ) at the base of the $\mathrm{H} \mathrm{CZ}$. Ca is supported at the base of the He CZ, but not at all $\left(g_{R A D}<<g\right)$ at the base of the H CZ. The rapid decrease of the surface $\mathrm{Ti}$ abundance following the peaking phase is due to mass loss draining the (now Ti-enriched) $\mathrm{CZ}$ from above.

It appears from Fig. 2 that at least for Ti, circulation does have an influence on surface abundances, as the $v_{e}=0$ and $50 \mathrm{~km} \mathrm{~s}^{-1}$ curves lie well below the 75 and $90 \mathrm{~km}^{-1}$ curves (although it can be verified that the curves converge again beyond about $10^{8} \mathrm{yrs}$ ). This is the exception rather than the rule. For most elements, little dependence on rotation rate is expected for most chemical species, in agreement with observations. This remains the case even when both meridional circulation and mass loss are included in the diffusion model.

\section{CHEMICAL SEPARATION IN ABOOTIS STARS}

The $\lambda$ Bootis stars are also main-sequence, Pop. I, non-magnetic chemically peculiar stars (Baschek \& Searle 1969; Gray 1988; Bohlender \& Landstreet 1990; Gerbaldi \& Faraggiana, these proceedings). They are rather rare objects (only a dozen or so $\lambda$ Bootis stars have been catalogued), and are restricted to the relatively narrow spectral type interval A0-F0 (Gray, 1988). This makes them very similar to the FmAm stars, except that their observed pattern of abundance anomalies is strikingly different. Most iron-peak elements are underabundant by factors of 3-10 (traditionally; recent work suggests as low as 50-100), while C, N, O and probably S are normal (Baschek \& Slettebak 1988; Venn \& Lambert 1990). Except for a possible lack of slow $\left(<50 \mathrm{~km} \mathrm{~s}^{-1}\right)$ rotators, their $v \cdot \sin i$ distribution appears compatible with normal stars in this spectral type interval, inasmuch as weight is to be attached to a distribution made up of only a dozen stars. A significant fraction of $\lambda$ Bootis have infrared excess (Sadakane \& Nishida 1986; see also King \& Patten 1992), and at least one exhibits absorption in the core of the Ca II K line (Gray, 1988).

\subsection{A diffusion-based model}

Because $\lambda$ Bootis stars are so similar to FmAm stars in most aspects other than their patterns of abundance anomalies, their inclusion within the general framework of diffusion models for CP stars may appear problematic. However, Michaud \& Charland (1986) showed that otherwise normal A and F stars undergoing mass loss at a rate of order $10^{-13} M_{\odot} y r^{-1}$ would over time develop the abundance signature of $\lambda$ Bootis stars. That this signature is so different from that of FmAm stars arises because the higher mass loss prevents the disappearance of the $\mathrm{He} \mathrm{CZ}$ (see Table 1), and the associated internal velocity field has time, over the main-sequence lifetime, to advect to the surface material previously depleted by chemical separation operating deep in the envelope.

Taken as it stood at the time of its inception, this mass loss-based diffusion model faces two possible difficulties: (1) the required abundance patterns take 
about $10^{9}$ yrs to materialize, thus restricting the $\lambda$ Bootis phenomenon to the end of the main-sequence phase for stars in this mass range, and (2) the effects of circulation are neglected. Since then, recent observational work has suggested that (1) may well be a serious problem (see Gray, 1988). It also generated two additional potential problems: (3) large underabundances (by factors of 50100 ) in general do not materialize (see Venn \& Lambert 1990), and (4) the restriction to a narrow spectral type interval demonstrated by Gray (1988) is hard to explain without introducing additional ad hoc assumptions.

A small set of solutions to chemical separation in the presence of meridional circulation within the framework of Michaud \& Charland's diffusion-based model for $\lambda$ Bootis stars (Charbonneau $1992 a, \S 3$ ) indicates that point (2) above may well be a critical problem. This latter issue is addressed in detail in Charbonneau (1992c); the following section present a sample of results from that paper.

\subsection{The influence of circulation}

Solutions to chemical separation in the presence of mass loss and circulation have been obtained using the finite element-based numerical scheme described in Charbonneau \& Michaud (1991) and Charbonneau (1992c). Using a $T_{\text {eff }}=8000$ $\mathrm{K}, \log g=4.3$ main sequence model and starting from an initial condition of homogeneous abundances throughout the envelope, the abundance evolution for two representative elements, $\mathrm{Ti}$ and $\mathrm{Ca}$, is followed over a time span of $10^{9}$ yrs. Figure 3 shows snapshots at $t=5 \times 10^{8}$ yrs of four solutions for $\mathrm{Ti}$, using various mass loss rates and equatorial rotational velocities. The variable $w$ is a normalized $\log (\Delta M / M)$, covering the interval $-11 \leqslant \log (\Delta M / M) \lessgtr-3$. The use of a regular mesh based on $w$ as a radial variable yields a higher resolution in the outer layers of the star. The angular variable is $\zeta=1-\cos \theta$ (so that $\zeta=0$ is the polar axis and $\zeta=1$ the equatorial plane). The gray scale codes $c(w, \zeta) / c_{0}$ logarithmically, where $c_{0}$ is the initial concentration.

Consider first the non-rotating model. The abundance distribution is spherically symmetric (i.e., no dependence on $\zeta$ ), since velocity fields associated with all transport mechanisms involved are themselves spherically symmetric. The underabundances having developped via settling below the depth where $g_{R A D}<g(w \leqslant 0.4)$ have by now been advected all the way into the CZ, leading to the appearance of underabundances in the $\mathrm{CZ}$ (dark gray). This is the essence of Michaud \& Charland's (1986) diffusion/mass loss model: underabundances develop first deep in the envelope, but are at later epochs advected to the surface by mass loss. In this two-steps process, the development of deep underabundances at early epochs of the evolution is critical.

Turn now to the rotating models. In the presence of meridional circulation, the evolution of internal abundances is totally different. Meridional circulation prevents the development of underabundances deep in the envelope, making it impossible for underabundances to materialize in the CZ at later epochs. This is clearly apparent on the remaining three solutions shown on Fig. 3. Note how the regions of underabundances present in the non-rotating model (black and dark gray) have been completely washed out in the rotating models, even at the relatively low rotation rate of $v_{e}=50 \mathrm{~km} \mathrm{~s}^{-1}$. Again, this is because meridional circulation velocities decrease much more slowly with depth than the velocities associated with chemical separation and/or mass loss. A search of the $\left[\dot{M}, v_{e}\right]$ parameter space reveals no region where generalized underabundances 

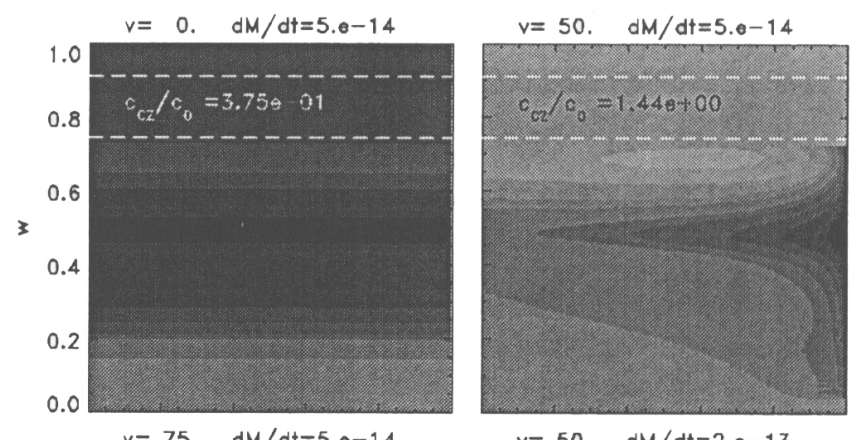

$t(y r s)=5.00 e+08$
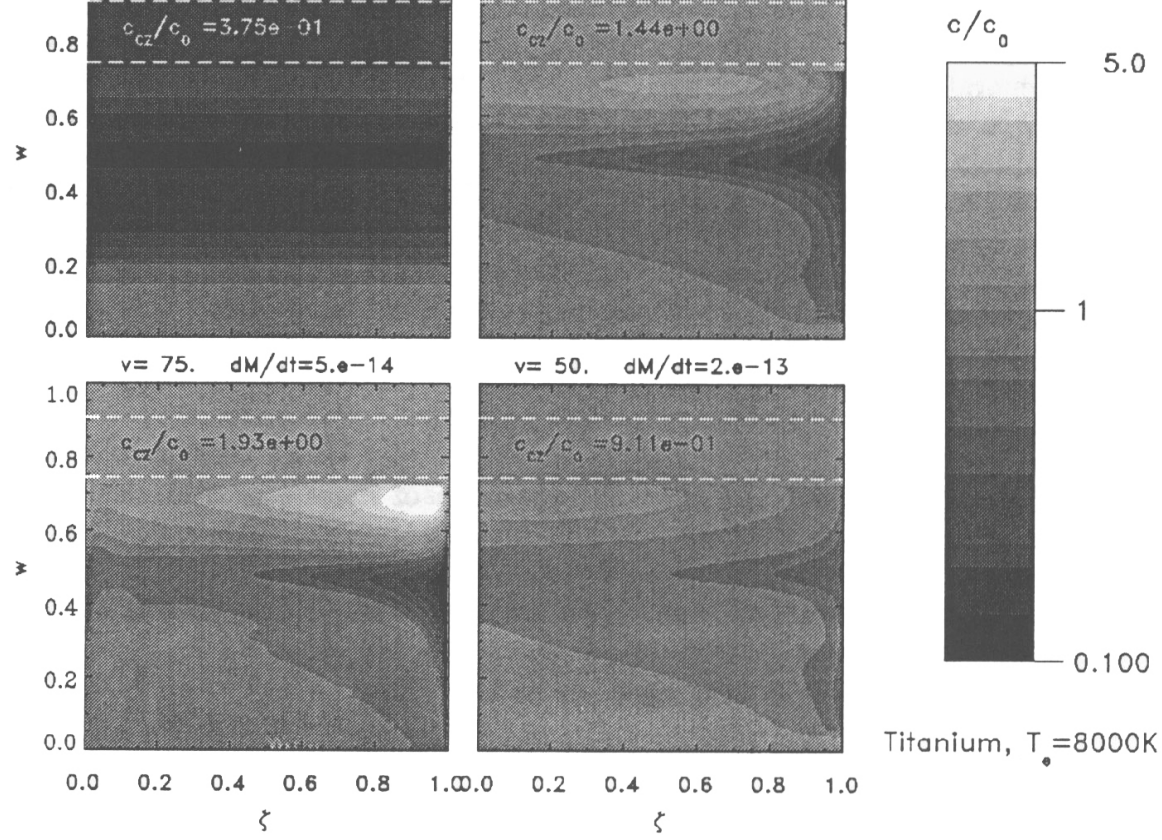

Titanium, $\mathrm{T}_{\bullet}=8000 \mathrm{~K}$

Figure 3 : Snapshot at $t=0.5$ Gyrs of the internal abundance distribution of $\mathrm{Ti}$ in four models with various equatorial rotational velocities and mass loss rates. The variable $w$ is a measure of depth ( $w=1$ corresponds to the surface), and $\zeta$ an angular variable ( $\zeta=0$ is the pole, $\zeta=1$ the equatorial plane). The dotted lines at $w \simeq 0.74$ and $w \simeq 0.9$ indicated the positions of the bases of the $\mathrm{He}$ and $\mathrm{H} \mathrm{CZs}$, respectively. The gray scale codes $c / c_{0}$ logarithmically, with black corresponding to underabundances, and white to overabundances. The inclusion of meridional circulation transport prevents the development of underabundances deep in the envelope, in turn making it impossible for underabundances to materialize at the surface at later epochs (see text).

materialize at any epoch of the evolution. Similar results have been obtained for $\mathrm{Ca}$ and $\mathrm{Sc}$, and in main-sequence models having $T_{\text {eff }}=7500 \mathrm{~K}$.

Figure 4(A) shows the corresponding time-evolution of the surface abundances. Similar solutions for calcium are shown on Figure 4(B). The solid lines correspond to the abundance evolution in a non-rotating model, as in the standard model of Michaud \& Charland (1986). Note again how significant surface underabundances no longer materialize when the additional mixing effects of rotationally-induced meridional circulation are taken into account. 

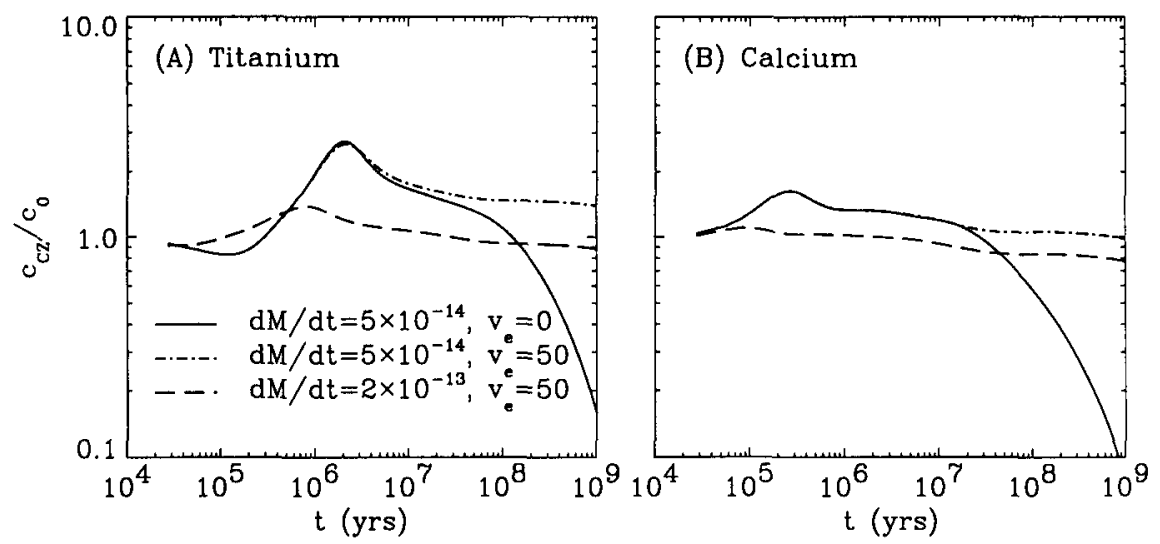

Figure 4: Surface abundance evolution of calcium and titanium in a $T_{\text {eff }}=8000 \mathrm{~K} \mathrm{\lambda Bootis} \mathrm{star} \mathrm{rotating} \mathrm{with} v_{e}=50 \mathrm{~km} \mathrm{~s}^{-1}$, for two values of the mass loss rate. Note how even at this relatively low rotation rate, the inclusion of transport by meridional circulation prevents the appearance of generalized underabundances at later epochs.

How can circulation have such drastic effects on the diffusion of metals in $\lambda$ Bootis stars, but not in FmAm stars (cf. $\oint 3.3$ above)? To understand this one must recall that within the framework of the diffusion model, the abundance signature of FmAm stars is governed mostly by chemical separation occurring immediately below the $\mathrm{H} \mathrm{CZ}$ (cf. Fig. 1 herein). In the Michaud \& Charland (1986) diffusion model for $\lambda$ Bootis stars, in constrast, chemical separation occurs only up to the base of the He CZ. More important, it is the development of underabundances deep in the envelope that is essential to the appearance of generalized heavy metal underabundances at later epochs. Now recall that for a given element and rotation rate, the ratio of diffusion to circulation velocities decreases sharply as one moves deeper and deeper in the radiative envelope. It is then not surprising to find that circulation plays a lesser role in the diffusion of metals in FmAm stars than in $\lambda$ Bootis stars.

These results, together with the recent discovery of a $\lambda B$ Bootis star in a very young $\mathrm{OB}$ association in Orion (Gray \& Corbally, these proceedings), indicate that the diffusion/mass loss model, at least in the form put forth by Michaud \& Charland (1986), must be abandoned.

\subsection{Another diffusion-based model}

Building on a suggestion by Venn \& Lambert (1990), Charbonneau (1991) showed that the $\lambda$ Bootis abundance signature would materialize in otherwise normal $\mathrm{A}$ and $\mathrm{F}$ stars accreting metal-depleted gas at a rate of order $10^{-13} M_{\odot} y r^{-1}$. Despite the preliminary nature of Charbonneau (1991)'s computations, the model seems quite succesful on a number of points: (1) the restriction to the spectral type interval A0-F0 is reproduced naturally, i.e., without adjustable parameters, (2) abundance anomalies can materialize within $10^{5}-10^{6} \mathrm{yrs}$, and (3) estimates of the effects of rotation suggests that meridional circulation does not obliterate the abundance anomalies. 
Some potential problems remain, however. A somewhat delicate fine tuning of the accretion rate appears to be required. Furthermore, the starting assumption of the model is that the (depleted) interstellar gas, previously depleted in metals following grain formation, is accreted onto the star without concomittant accretion of the grains. This does not appear obvious. Small grains indeed tend to dynamically decouple from the surrounding gas in conditions pertaining to the circumstellar environment of cool evolved stars (see, e.g., MacGregor \& Stencel 1992). Around an A-star, however, the non-neglibible UV flux will lead to photoionization and subsequent electrical charge buildup on the grains, drastically increasing the coupling with the surrounding gas. While it may well be that separation of gas and grains still occurs under these conditions at least over part of the grain size spectrum, it must be emphasized that at this juncture, the "gas without grains" accretion hypothesis is completely ad hoc.

Should the accretion of gas without grains be shown to be possible, it is also important to realize that this is only the beginning of the story. Accretion or not, chemical separation still occurs in the radiative envelope. It does not go away or disappear just because accretion is turned on. The evolution of observed abundances is determined by both the accreting flow at the surface and chemical separation occuring at the base of the $\mathrm{CZ}$ (see, in a different but related context, Proffitt \& Michaud 1989). Of course for a high enough accretion rate the effects of chemical separation will be washed out, but this is not the case at the rate of $10^{-13} M_{\odot} y r^{-1}$ suggested by the model of Charbonneau (1991). This certainly justifies performing more detailed particle transport calculations within the diffusion/accretion framework.

\section{CONCLUSIONS}

The results presented above illustrate situations where rotationally-induced meridional circulation plays an important role in the abundance evolution (He settling in A stars and evolution of metal abundances in $\lambda$ Bootis stars), as well as a situation where it does not (evolution of metal abundances in FmAm stars). That circulation-mediated particle transport, for a given rotation rate, can be important in some instances and not in others should serve as a reminder that the interaction of meridional circulation with other particle transport processes is complex and often subtle. Overly enthusiastic extrapolation of a given set of results outside the parameter ranges covered by the computations remains a very risky procedure.

This is perhaps the proper place to say a few words about rotationallyinduced turbulence (see, e.g., Zahn 1987). Turbulence impedes chemical separation (Schatzman 1969), and under certain circumstances it may also impede transport by meridional circulation (Chaboyer \& Zahn 1992; Charbonneau 1992b). The number of instabilities, driven and/or controlled by rotation, potentially leading to turbulence in radiative zones of rotating stars abound, and there are no reasons whatsoever to believe that large-scale flows in stars should be strictly laminar. But here the efforts of modellers are plagued by the lack of a quantitative model for turbulent particle transport in radiative zones. Rotationally-induced turbulence has been repeatedly advocated as being the agent governing the evolution of light element abundances in F and $G$ stars, as well as in the Sun (e.g. Schatzman 1977; Baglin, Morel \& Schatzman 1985; Vauclair 1988; Charbonnel et al. 1992). But unfortunately, results obtained by 
these (and other) authors tend to depend rather sensitively on the form of their assumed parameterization of turbulent transport.

Again perhaps the most constructive approach in this context is to use observed abundances in conjunction with particle transport models to help constrain parameterizations of turbulent transport (see e.g. Vauclair et al. 1978; also Pinsonneault et al. 1989 for a large-scale example). Within the diffusion model for FmAm stars, Charbonneau \& Michaud $(1991, \$ 5)$ found that at least in stars rotating with $v_{s} \leqslant 100 \mathrm{~km} \mathrm{~s}^{-1}$, very little turbulence can be tolerated in the upper part of the radiative envelope. This result turns out to be almost independent of the adopted parameterization for turbulent mixing.

I would like to conclude with a short list of interesting observational and modelling "problems". These may perhaps not be the most fundamental issues to be adressed in their respective contexts, yet they represent questions that, in my opinion, can be succesfully answered in the near future, given the current status of abundance observations and modelling.

\subsection{Observations}

While my understanding of observational matters is too limited to make confident claims as to what is possible and what is not, the following observational "tasks" do not appear to me unreasonable: (1) To determine abundance trends for a few elements across the width of the main-sequence, for both CP stars and "normal" stars. Work along these line is already well under way (e.g., Holweger et al. 1986a,b; Lemke 1989; Berthet 1992; Holweger, this conference; and references therein), and should be energetically pursued. (2) To observe selected FmAm stars for evidence of X-ray emission. If the winds in these stars are thermally driven by a hot corona, the associated X-ray emission may well provide the only direct detection of such winds; upper limits on mass loss rates in A stars obtained from the study of radio emission or spectral line asymmetries (e.g. Brown et al. 1990; Lanz \& Catala 1992) are orders of magnitude above the rates required by the diffusion model for FmAm stars. (3) To observe selected $\lambda$ Bootis stars for evidence of accretion.

\subsection{Modelling}

As an increasingly accurate picture of chemically peculiar stars is being drawn observationally across the width of the main-sequence, detailed diffusion calculations in evolutionary models for normal, FmAm, and $\lambda$ Bootis stars are increasingly needed. In the same vein, detailed accretion/diffusion calculations for $\lambda$ Bootis stars should be carried out, if the predictions of the accretion hypothesis are to become quantitative enough to allow a detailed comparison with observations.

Along different lines, it would be most interesting to put the mass loss hypothesis on firmer physical grounds, as it currently remains an ad hoc hypothesis. The construction of thermally-driven wind models applicable to $\mathrm{A}$ and $\mathrm{F}$ stars would be a good starting point. Likewise, simple grain/gas accretion calculations in a circumstellar environment characteristic of $\mathrm{A}$ stars could indicate whether the accretion hypothesis for $\lambda$ Bootis stars is physically realistic. More specifically, one should investigate whether or not it is possible 
to set up an accretion flow around an A-type star such that gas can be accreted without concomittant accretion of grains.

Finally, it would be interesting to test J.-P. Zahn's (1992) meridional circulation formulation in the context of the diffusion model for FmAm (and $\mathrm{HgMn}$ ) stars. Zahn argues that the good agreement obtained within the diffusion model for FmAm and HgMn stars using Tassoul \& Tassoul (1982)'s meridional circulation solutions is a mere coincidence. While this cannot be strictly ruled out, it appears (to this author at least) that until an alternative model with equal or superior quantitative predictive power is developed, the diffusion-based model for CP stars, as it currently stands, is to be preferred.

\section{ACKNOWLEDGEMENTS}

I wish to thank the organizers of the IAU Colloquium 138 for their invitation to attend the meeting, and V. Hansteen for his critical reading of the first draft of this paper. Computations reported here were carried out on the Cray Y-MP/864 of the National Center for Atmospheric Research.

\section{References}

Abt, H. A., \& Levy, S. G. 1985, Ap. J. Suppl., 59, 229

Baglin, A., Morel, P.J., \& Schatzman, E. 1985, Astr. Ap., 149, 309

Baschek, B., \& Searle, S. 1969, Ap. J., 155, 537

Baschek, B., \& Slettebak, A. 1988, Astr. Ap., 207, 112

Berthet, S. 1992, Astr. Ap., 253, 451

Bohlender, D.A., \& Landstreet, J.D. 1990, M. N. R. A. S., 247, 606

Brown, A., Vealé, A., Judge, P., Bookbinder, J. A., \& Hubeny, I. 1990, Ap. J., 361,220

Burkhart, C., \& Coupry, M.-F. 1991, Astr. Ap., 249, 205

Cayrel, R., Burkhart, C., \& Van't Veer, C. 1991, in IAU symp. 145, Evolution of Stars: The Photospheric Abundance Connection, eds. G. Michaud \& A. Tutukov, 99

Chaboyer, B., \& Zahn, J.-P. 1992, Astr. Ap., 253, 173

Charbonneau, P. 1991, Ap. J., 372, L33

Charbonneau, P. 1992a, J. Roy. Astron. Soc. Canada, 86, 31

Charbonneau, P. 1992b, Astr. Ap., 259, 134

Charbonneau, P. 1992c, preprint

Charbonneau, P., \& Michaud, G. 1988, Ap. J., 327, 809

Charbonneau, P., \& Michaud, G. 1991, Ap. J., 370, 693

Charbonnel, C., Vauclair, S., \& Zahn, J.-P. 1992, Astr. Ap., 255, 191

Gray, R. O. 1988, Astron. J., 95, 220

Holweger, H., Gigas, D., \& Steffen, M. 1986a, Astr. Ap., 155, 58

Holweger, H., Steffen, M., \& Gigas, D. 1986b, Astr. Ap., 163, 333

King, J.R., \& Patten, B.M. 1992, M. N. R. A. S., 256, 571

Lanz, T., \& Catala, C. 1992, Astr. Ap., 257, 663

Latour, J., Toomre, J., \& Zahn, J.-P. 1981, Ap. J., 248, 1081

Lemke, M. 1989, Astr. Ap., 225, 125

MacGregor, K. B., \& Stencel, R. 1992, Ap. J., 397, in press

Michaud, G. 1982, Ap. J., 258, 349

Michaud, G. 1987, Phys. Scripta, 36, 112 
Michaud, G., \& Charland, Y. 1986, Ap. J., 311, 326

Michaud, G., \& Charland, Y., Vauclair, S., \& Vauclair, G. 1976, Ap. J., 210, 447

Michaud, G., Tarasick, D., Charland, Y., \& Pelletier, C. 1983, Ap. J., 269, 239

Pinsonneault, M.H., Kawaler, S.D., Sofia, S., \& Demarque, P. 1989, Ap. J., 338, 424

Preston, G. W. 1974, Ann. Rev. Astr. Ap., 12, 257

Proffitt, C.R., \& Michaud, G. 1989, Ap. J., 345, 998

Schatzman, E. 1969, Astr. Ap., 3, 331

Schatzman, E. 1977, Astr. Ap., 56, 211

Sadakane, K., \& Nishida, M. 1986, PASP, 98, 685

Sakurai, T. 1991, M. N. R. A. S., 248, 457

Sofia, S., \& Chan, K.L. 1984, Ap. J., 282, 550

Tassoul, J.-L. 1978, Theory of Rotating Stars (Princeton: Princeton University Press)

Tassoul, J.-L. 1990, in Angular Momentum and Mass Loss for Hot Stars, eds. L. A. Willson \& R. Stalio (Kluwer), 7

Tassoul, J.-L., and Tassoul, M. 1982, Ap. J. Suppl., 49, 317

Vauclair, G., Vauclair, S., \& Michaud, G. 1978, Ap. J., 223, 920

Vauclair, S. 1988, Ap. J., 335, 971

Venn, K. A., \& Lambert, D. L. 1990, Ap. J., 363, 234

Zahn, J.-P. 1987, in The Internal Solar Angular Velocity, B. Durney \& S. Sofia (Dordrecht: Reidel), p. 201

Zahn, J.-P. 1992, submitted to Astron. Ap. 